\title{
Neuro-Link, a Computer-Assisted Database for Head Injury in Intensive Care
}

\author{
G. Citerio $^{1}$, N. Stocchetti ${ }^{2}$, M. Cormio ${ }^{1}$, and L. Beretta ${ }^{3}$ \\ ${ }^{1}$ Rianimazione, H San Gerardo, Monza (Mi) \\ ${ }^{2}$ Terapia Intensiva Neurochirurgica, IRCCS H Policlinico, Milano \\ ${ }^{3}$ Neurorianimazione, IRCCS H San Raffaele, Milano Italy
}

\begin{abstract}
Summary
Reliable information is vital for clinical trials, so we developed a database, for head trauma victims admitted to neuro-intensive care units (NICU). This database, first step in a sequential project, comprises 176 selected fields mainly focused on the early post-traumatic phase and has a user-friendly computerized interface. The software was tested for a trimester in 18 Italian neuro-intensive care units.

The paper describes the main features of the database, the results of a three months' data collection test, its limitations and its potential improvements.

A description of the database fields and a brief summary of the 282 patients included so far are also presented.
\end{abstract}

Keywords: Head injury; database; intensive care; outcome.

\section{Introduction}

Over recent years, many databases on head injured patients have been developed. Some have been designed for data collection during drug clinical trials, others to investigate epidemiology, severity, clinical features and outcome. In the USA, during the 80 's, the "Traumatic Coma Data Bank" (TCDB), a collection of prospective observational data from head injured patients, was set up and promoted by the National Institute of Neurological Disorders and Stroke (NINDS) [3]. The TCDB was intended to study the neurological course and to evaluate the outcome determinants of patients with traumatic coma. This has resulted in more than 30 papers, covering many aspects of traumatic brain injury. In the 70's, the International Data Bank, with the aim of investigating the prognosis of coma, entered information on 700 head trauma patients collected in the UK, Netherlands and USA [4].

More recently the European Brain Injury Consortium, EBIC, [9] ran a three-month observational data collection study, from February to April 1995, in 67
European Neurosurgical Centers [8]. Information was gathered on 1005 severe and mild head injured patients.

During pharmacological trials on head injury [6] a huge amount of clinical information has been coded and assembled. It was not unusual to spend from 40 to 80 hours completing the forms required by that trial.

Data on cases not enrolled in clinical trials, however, are usually scarce and not codified. It is clearly not practical to extend the huge resources required by drug trials to all patients but it did seem feasible to exploit the experience gained in such studies to plan a new clinically useful data collection.

In 1996, three neuro-intensive care units (NICU) in the Milan metropolitan area devised a database, named "Neuro-Link", for simple, computer-assisted collection of the main information on head injury. The aim was to provide a picture of their clinical practice, to provide a tool for quality assurance and for registry information on outcomes and complications. Moreover, the database was considered as a starting point for planning extended data collection and clinical studies.

This paper describes the design of "Neuro-Link", summarizing our experience with the use and implementation of this database and discussing its potential for research and clinical use. Preliminary data on the cohort recruited are described.

\section{Methods and Patients}

Design of the Database

The data collection form was designed for use with a computer interface and is composed of six different computer screen layouts. They cover: 
1. General data

2. Pre and admission data

3. Secondary insults and complications

4. Computed tomography (CT) scan data

5. Monitoring information

6. Discharge and outcome information

Details on the information collected are presented in the appendix. Each page is connected to two additional sections, one to assist filling in the database process and the other allowing the addition of free text to the coded information. The Help section, in which three elements of information are condensed, offers assistance.

First, the general purpose and design of every page is specified. Second, every field is defined and coded, offering a complete on-line codebook. Third, practical examples are included, describing the most common situations and mistakes.

A Steering Committee of the authors supervised all the development phases, discussing all details from the ideation to the completion of the data collection and analysis.

\section{Inclusion and Exclusion Criteria}

Inclusion criteria in the database were very wide so as to describe clinical reality as fully as possible. All head injured patients, regardless of Glasgow Coma Score and age, requiring intensive care during the first 24 hours post-trauma were eligible. Since many polytrauma patients are admitted to the intensive care unit for extracranial injuries associated with trivial head injuries, only cases in whom the head injury was the real ICU admission reason were included.

Gunshot wounds and spinal cord injuries were excluded, the former because the mechanism of missile injury is special and the latter because the presence of a spinal cord injury has an overwhelming effect on disability. Patients who failed to respond to non-surgical resuscitation (patient dead on arrival) and were therefore not admitted to the NICU were not inserted in the database.

\section{Software Interface}

The Neuro-Link software was developed by two of the authors (MC and GC) using File Maker Pro, version 4 (Claris Corporation, Santa Clara, CA, USA). The latest Neuro-Link version, released in January 1998, is a run-time version produced using File Maker Pro SDK (Claris Solution Alliance, Claris Corporation, Santa Clara, CA, USA) running on Macintosh or Window-based computers.

The database captures 176 information items. Each group of information is presented on a single screen, for ease of completion. Buttons are used to allow simple "navigation" through the different parts of the program.

A manual was written, containing definitions and clinical examples for every field. The manual has been incorporated in the software and printed as a booklet. A Web page was published on Internet, so as to "publicize" the project and facilitate communications through e-mail.

A pilot data collection study started in January 1997 in three units in Milan. After this test phase, the data collection was extended to other NICU in the country, known for their interest in neurotrauma care.

\section{Training}

Twenty Italian NICU, listed in the acknowledgements section, sent at least one representative to a teaching course held in Monza, where the general design and detailed aspects of the database were described. "Virtual" cases (i.e. fictitious patients to be entered in the database) were presented and discussed so as to avoid misinterpretation of the data collection and of the software.

\section{Data Collection and Funding}

Patients' enrolment started on September 1st 1997 in 18 Italian NICU which, after the training course, had expressed the desire to participate. Data collection lasted three months and ended with a six-month follow-up. The Neuro-Link software was released free to all the participating centers.

A 24-hour help line (by phone or E-mail), based in the coordinating centre at Monza ICU, was available during the data collection period. Electronic transfer via Internet was planned to guarantee fast data flow from peripheral centers. Data were returned to the coordinating center at the end of the enrolment period, together with two CT scan copies codified for each patient. Before starting the data collection, an authorization was requested and obtained by the Italian "Autorità Garante della Privacy", a Ministerial office deputed to solve and control privacy issues. Data were transferred protected by a password. The final file, composed by the data received by all the centers, was always protected by password. The final version of the database contains personal data (name, address, phone number) crypted.

A neuroradiologist, independent of the study, evaluated the CT scans, to verify the data inserted and for additional research purposes. Only as examples, analysing the first CT scan, postraumatic subarachnoid hemorrhage was recorded in 44 of the cases and was identified by the independent neuroradiologist in $59 \%$ of the cases. A higher agreement was noted in perimesencephalic cisterns status; were described as "compressed" in $53 \%$ vs $46 \%$ respectively in the centers and central reading. Shift absence was coded in $67 \%$ vs $63 \%$ of the CT scans. More detailed information on centralized scans reading will be reported elsewhere.

Incoming data were carefully reviewed during and after the study period. After a first review, queries were submitted to the centers to clarify information, to overcome inconsistencies, and to obtain missing data. Queries were sent by E-mail or fax and were followed by a phone call. All the patient outcomes were collected six months after injury using the Glasgow Outcome Scale (GOS).

The entire project was conducted on a limited budget. Funding was provided by "Il Raggio Verde", an Italian no-profit association for NeuroICU research.

\section{Statistics}

The methods used are largely descriptive. All summary data are expressed as mean \pm standard deviation (SD). Means were compared by analysis of variance. Differences in proportions were analyzed by the Chi-square test. A level of $\mathrm{p}<0.05$ was considered significant.

\section{Results}

\section{Training}

All participants who attended the training course were able to input the "virtual" cases correctly in the computerized database.

\section{Data Flow and Quality}

Only 11 centers sent data through the Net. Five others entered the data in their computers and sent diskettes by mail; the last two mailed printed matter.

Data collection ended on December 1st, 1997, and 
the last forms were obtained in February 1998. Several requests and reminders were sent to each center to obtain patients' data and missing information. The last follow-ups were in October 1998. As part of the quality assurance, we developed a structured search that led to the elimination of logical inconsistencies. In Autumn 1998 the information still missing was requested and a list of inconsistencies was sent for clarification.

We identified a set of 39 fields considered essential for classifying the patient. They are absolutely not exhaustive but their absence does not permit one to define the severity of head trauma and secondary insults. They are to be intended as "mandatory" fields. Out of a total of 10.998 essential data expected, only 195 $(1.7 \%)$ were still missing at the end of the revision phase.

\section{Enrolment Rate}

Data from 18 centers were collected for a total of 282 patients. Table 1 shows the number of head injury cases admitted to each ICU. The recruitment rate per center varied widely, from 1 to 40 patients. Five centers enrolled more than 20 patients in the study period, eight admitted from 10 to 20 patients and five had only a limited number of patients, i.e. less than 10 per trimester.

\section{Demographics}

The mean age of the study population was $39.9 \pm 21$ years, median 33 years, range between 3 months and

Table 1. Neurointensive Care Units and Patients Enrolled (Total of 282)

\begin{tabular}{ll}
\hline ICU & Number of patients \\
\hline Treviso & 40 \\
Milano Niguarda & 32 \\
Monza & 29 \\
Ancona & 23 \\
Milano Policlinico & 20 \\
Torino CTO & 17 \\
Pavia II & 16 \\
Bologna Bellaria & 15 \\
Roma Gemelli & 14 \\
Lecco & 13 \\
Milano San Raffaele & 13 \\
Vicenza & 13 \\
Trieste & 12 \\
Cesena & 9 \\
Varese & 7 \\
Brescia II & 6 \\
Genova Galliera & 2 \\
Sondalo & 1 \\
\hline
\end{tabular}

86 years. Patients were predominantly males $(73 \%)$ and the main cause of injury was road traffic accidents (73\% including pedestrians and vehicle occupants), followed by household accidents $(12 \%)$. Less frequent were sport trauma $(2 \%)$, work accidents $(6 \%)$ and the remaining $7 \%$ include the other unclassified aetiologies.

\section{Referral to NICU}

One hundred and twenty patients $(42 \%)$ were admitted directly to a "study" hospital with NICU and neurosurgical facilities. The other 58\% (162 patients) were transferred from peripheral hospitals. Patients admitted directly from the accident site to a neurotrauma hospital took $79 \pm 149$ minutes to reach the Emergency Room. In the transferred cohort, the first Emergency Room was reached in $59 \pm 137$ minutes and the patient arrived later at the neurotrauma hospital (mean $300 \pm 254$ minutes).

\section{Extracranial Injuries}

Only lesions actually requiring hospitalisation were recorded in the database. Table 2 indicates the frequency of each coded extracranial lesion. At least one extracranial lesion was present in 146 patients $(52 \%)$. Haemorrhage, defined as the requirement for more than two units of packed red blood cells in the first 24 hours, was the most frequent associated clinical condition, followed by major chest trauma and pneumothorax which required drainage.

Eleven percent (31 patients) underwent extracranial surgery during the first day post-trauma. Extracranial surgery requirements were no different for directly admitted and transferred patients in (respectively 14\% and $9 \%$ ).

Table 2. Extracranial Lesion

\begin{tabular}{ll}
\hline Extracranial lesion & Patients \\
\hline Haemorrhage & 55 \\
Major chest trauma & 44 \\
Pneumothorax & 26 \\
Major abdominal injury & 19 \\
Major limb injury & 61 \\
Major facial injury & 55 \\
Major pelvic injury & 17 \\
Spinal amyelic lesion & 6 \\
\hline
\end{tabular}

All major lesions required hospital admission by themselves. 


\section{Clinical Evaluation}

Neurological evaluation was accomplished combined the Glasgow Coma Score (GCS) and evaluation of pupils at three time points: before reaching the neurosurgical Hospital, at Neurosurgical Hospital admission and post-stabilization (see appendix). Sedation was frequently used (respectively $51 \%$ and $40 \%$ of the transferred and directly admitted patients were properly sedated) but evaluation "windows" were frequently opened. GCS was available for 150 cases $(59 \%)$ before neurosurgical hospital admission, 248 patients $(89 \%)$ at Neurosurgical Hospital admission and $229(82 \%)$ post-stabilization. Motor GCS was untestable because of curarisation in 32 cases at Neurosurgical Hospital admission and in 11 post-stabilization.

The proportion of patients with GCS $\leq 8$ varied from $59 \%$ of the pre-Neurosurgical Hospital scores to $69 \%$ at Neurosurgical Hospital admission and from 26 to $29 \%$ patients were classified as obeying commands at the three time points. Pupil reactivity at the three time points was tested in $259(90 \%), 275(96 \%)$ and 280 $(99 \%)$ cases. Signs of transtentorial herniation, i.e. one unreactive dilated pupil, and of inadequate cerebral perfusion, i.e. both pupils dilated, were present in the same percentage at all three times (one fixed dilated pupils $10-14 \%$, both fixed dilated pupils $9-13 \%$ ).

\section{Admission CT Scans}

CT scan was performed earlier in the patients admitted directly to a hospital with neurosurgery, where this facility is available round the clock. The mean interval between trauma and CT was $84 \pm 236$ minutes. In secondarily admitted patients this interval was longer, $194 \pm 236$ minutes.

The most frequent intracranial lesion seen on the first CT scan was an intracerebral mass $(31 \%)$, followed by subdural haematoma (28\%) and extradural haematoma (16\%). One quarter of the first CT scans showed no mass. Lesion volumes were calculated using Cavalieri's direct estimator [2]. On the first CT scan with at least one hyperintense lesion $(210,74 \%$ of the total scan), mean lesion volume was $38 \pm 45 \mathrm{ml}$ (median $20 \mathrm{ml}$ ). A lesion larger than $25 \mathrm{ml}$ was identified in $101 / 210$ positive scans ( $48 \%$ ).

Subarachnoid haemorrhage was recorded in 138 patients $(48 \%)$, intraventricular haemorrhage in 27 $(9 \%)$, pneumocephalus in $44(15 \%)$. Indirect signs of
Table 3. Secondary Insults in the First 24 Hours

\begin{tabular}{lcll}
\hline & Clinical & Definite & None \\
\hline Hypoxia & $35(12 \%)$ & $41(15 \%)$ & $204(73 \%)$ \\
Hypotension & $9(3 \%)$ & $62(22 \%)$ & $209(75 \%)$ \\
\hline
\end{tabular}

raised intracranial pressure, i.e. shift $\geq 5 \mathrm{~mm}$ and compressed perimesencephalic cisterns, were present in respectively $27 \%$ and $53 \%$ of the "study" population.

\section{Intracranial Surgery in the First 24 Hours}

In the first 24 hours, an intracranial intervention other than insertion of an intracranial pressure (ICP) system was done in 115 cases (32\%). An ICP device was inserted as the sole neurosurgical procedure in 52 patients $(18 \%)$. A total of 99 ICP devices, considering the cases in which it was the only procedure together with the operated cases, were placed in the first day post-trauma.

Surgery consisted of different combinations of the following possibilities: extradural haematoma evacuation (48 cases, $42 \%$ of the operated cases), subdural haematoma removal (59 cases, 51\%) and intracerebral clot evacuation (34 patients, $30 \%$ ).

\section{Secondary Insults}

Secondary insults occurrence are listed in Table 3. In the first 24 hours post-trauma, an hypoxic episode, i.e. $\mathrm{PaO}_{2}<60 \mathrm{mmHg}$ or $\mathrm{SpO}_{2}<90 \%$, was recorded in $27 \%$ of the population (76 patients). Hypotension, defined as systolic blood pressure (BP) $<95 \mathrm{mmHg}$, was reported in 71 patients $(25 \%)$. Mean systolic BP at neurosurgical hospital admission was $126 \pm 31 \mathrm{mmHg}$ (median $130 \mathrm{mmHg}$ ). At the same time, a quarter of this population had a systolic BP lower than $110 \mathrm{mmHg}$. Patients transferred from referral hospital were more frequently intubated $(88 \%)$ than cases directly admitted to neurosurgical hospitals (59\%). This difference was significant $(<0.0001)$.

\section{Monitoring}

A total of 121 patients were monitored with an ICP device. An ICP monitoring device was positioned in 97 $(52 \%)$ of 186 patients with a GCS $\leq 8$ at neurosurgical hospital admission. The most frequently used ICP device was a ventricular catheter (52 patients, $41 \%$ of 
the monitored cohort), followed by parenchymal (48, $38 \%)$ and subdural systems (26, 21\%). ICP monitoring was complicated by five small, non-surgical haemorrhages ( $4 \%$ ) and four cases of ventriculitis (3\%). Jugular bulb oxygen saturation $\left(\mathrm{SjO}_{2}\right)$ was measured frequently in patients with GCS greater or less than 8 (106 patients). Monitoring was done with standard intravascular catheters in 74 cases $(70 \%)$ and with fiber optic devices in $32(30 \%)$. This monitoring was used in $87 / 186$ GCS $\leq 8(47 \%)$ and in 18 patients not in coma. $\mathrm{SjO}_{2}$ was monitored in association with ICP in 91 patients, 77 of them in coma (admission GCS $\leq 8$ ). $\mathrm{SjO}_{2}$ was the only neuromonitoring in 15 patients, 10 of them with an admission GCS $\leq 8$.

\section{Complications in ICU}

Detailed definitions of the complications are available in the appendix. The most frequent intracranial complications were raised ICP (67/97 patients), defined as ICP $>20 \mathrm{mmHg}$ for at least 15 minutes, and intractable ICP, defined as ICP $>30 \mathrm{mmHg}$ for more than 30 minutes despite maximal therapy (32/97), followed by delayed haematoma (30/278).

Life-threatening extracranial complications were also frequent: respiratory (46 patients) and cardiovascular insufficiencies (51 patients). Pneumonia was recorded in 55 patients $(20 \%)$. Metabolic derangements included hyponatremia (46 patients) and hyperglycemia (77 patients).

\section{Six Month Outcome}

Six month follow-up was obtained from all centers for almost all the patients. Follow-ups arrived at the co-ordinating center in September 1998. Only a very small percentage $(4 \%)$ of the total population was lost to follow-up. Six-months mortality was $34 \%$, unfavourable outcome (i.e. vegetative plus severe disability) was $15 \%$ and favourable outcomes (i.e. good outcome and moderate disability) were 51\% (Table 4).

Analysing only severe head injured patients, limiting

Table 4

\begin{tabular}{lc}
\hline Glasgow Outcome Score (GOS) & Number of patients \\
\hline 1 (dead) & $92(33.6 \%)$ \\
2 (vegetative state) & $10(3.6 \%)$ \\
3 (severe disability) & $32(11.7 \%)$ \\
4 (moderate disability) & $33(12 \%)$ \\
5 (good outcome) & $107(39.1 \%)$ \\
Total Outcomes & $274(96 \%)$ \\
\hline
\end{tabular}

the outcome analysis only to patients in coma at NSHA arrival (GCS $\leq 8)$, at six months mortality was $44 \%$, unfavourable outcome was $16 \%$ and favourable outcomes were $40 \%$.

\section{Discussion}

Data from large samples may provide useful information on many aspects of head injury care, provided the data are of good quality. The quality, especially in multicenter, low-cost studies, can probably be improved, as described here, by using a computerassisted system. Our computerized database was, in fact, designed to limit input errors, to offer on-line help and to facilitate data audit and analysis.

Before this collection, no reliable, codified, controlled data were available in Italy about the severity and number of head trauma cases admitted to ICU.

This database was designed and developed by clinicians for clinical use. It was therefore meant to be simple, fast and user-friendly as far as possible. The data obtained regard an unselected cohort of head trauma victims, admitted to 18 Italian NICU. Entry criteria were very wide, compared with clinical drug trials. We were thus able to assemble a huge amount of information, with extremely limited cost, thanks purely to the authors and participants commitment.

More than $98 \%$ of the information required for classifying the patients was collected. Unlike in drug trials, restricted personnel and funding meant that computer data and the original hospital records were not checked to confirm the accuracy of the information. However, the coherence and consistency of the findings suggest the data are credible.

Demographics overlap the data presented in previous series. Patients are mostly young males, admitted to hospitals mainly after road traffic accidents.

Neurological data, i.e. GCS and pupil reactivity, essential for grading the severity of head injury were recorded in a very high percentage, indicating the use of sedation "windows" for evaluating patients. About a quarter of the cohort was not in coma, since they obeyed commands. Differences in entry criteria mean that the populations included in pharmacological trials and in other previous data collection, such as the TCDB, are not perfectly comparable.

Patients who arrived directly in a Neurosurgery hospital had a CT scan one and a half hours after trauma and transferred patients after three hours. These intervals between trauma and CT are long 
compared with European data, provided by the EBIC survey. Considering the widespread availability of CT scan facilities in Italy, this delay in intracranial diagnosis is extremely long. In many regions of Italy the health care system is not adequately organised to treat severely head injured patients and needs to be reviewed as soon as possible. The collected information, associated with an effort of diffusing treatment recommendations through out our country, can contribute to support and motivate insistence on new organization and resources.

In the last decades CT scan classification has evolved. We preferred to collect raw data, allowing us to describe CT scans with the current classification system [7] and, if the CT classification changes, we can re-classify our population. Moreover, although in the last ten years Neurosurgeons have described traumatic lesions according to the TCDB classification, which requires calculation of the mass lesion, there is no uniform method for this calculation. We used Cavalieri's direct estimator because it is easy to use after a short training. The Neuro-link software includes an integrated calculating system, which, with only limited information, establishes the mass lesion volume of the two largest lesions. We selected and classified the first and the worst CT scans obtained in the first two weeks after injury, for a better description of the course of the mass lesion and a precise description of the patient.

Frequencies of secondary insults are similar to previous series, such as the TCDB and EBIC surveys. Hypoxia is a frequent early insult, recorded in more than a quarter of the cohort. There was also a high incidence of hypotension, as in the European survey. Physiological parameters as BP and blood gas tension were similar in transferred and directly admitted patients. Long delays in transfer apparently had no negative influence on physiological parameters on arrival at a Neurosurgical hospital.

Urgent intracranial surgery was required by one third of the cohort and an extracranial operation was needed in $11 \%$.

Intracranial pressure was monitored in about half the population in coma. An ICP device was not used in some cases due to advanced age and/or poor clinical condition. Intracranial hypertension was frequent.

Jugular saturation was monitored more than ICP. Curiously, 15 patients had a jugular catheter as sole neuromonitoring device. This reflects a paradox of the Italian situation, where intensivists rather than neurosurgeons often have to take care of neurotrauma patients doing the best they can. Then too, neurosurgeons are not always involved in immediate trauma victim care.

The high rates of extracranial complications highlights the need for a combined approach, intensivist plus neurosurgeon, and different specialities must work hand in hand to help the patient reach a better outcome.

Six-month outcome was obtained in more patients than in previous series. Distribution between GOS classes reflects the known pattern for this population, with a higher proportion of good outcomes.

Data flow from centers was more difficult than expected. Neuro-link was designed to be simple and user-friendly, and was backed by training, on-line help, and a hot line. Incoming data were carefully reviewed during and after the study period. Nevertheless data were often missed and a number of inconsistencies were noted. Therefore a meticulous, time and laborconsuming audit of data was still necessary.

The net benefit of this computer-based system for data collection is, therefore, still not clear. Printed data had a higher rate of interpretation errors than data exchanged on magnetic supports. Multiple choices, with precoded fields, limited the possibility of errors in inputting data and make data management easier.

A second version of the Neuro-link software has been developed. It builds on the experience of this data collection, and has enhanced features for assisted data entry and for limiting inconsistencies and missing fields. Insertion of information not pre-coded is not allowed. The aim of this second release is to document population information during randomised clinical trials we are planning for the future associated with an extended report of the clinical events during the ICU stay. Data set on the pre-hospital phase have been reduced, limiting information at the identification of secondary insults that could affect the final outcome. A larger set of data is collected during the first week of ICU stay. We inserted data not only on the used monitoring modality but also on the results of the physiological collected information, i.e. intracranial pressure and cerebral perfusion pressure data.

We are planning future multicentric, prospective studies on head trauma victims in selected ICU which participated in the first co-operative phase.

It is not likely that any computer-based system will ever replace personal commitment and clinical observation, but new tools may reduce the burden of data collection and audit. 


\section{Acknowledgements}

We are extremely grateful to all the investigators, indicated in brackets, who co-operated in the Neuro-link data collection in the following 18 Italian neuro intensive care units: Ancona Torrette (Dr. Bini), Bologna Bellaria (Dr. Zanello), Brescia Civili $2^{\text {a }}$ Rianimazione (Dr. Febbrari), Cesena Bufalini (Dr. Nanni), Genova Galliera (Dr. Silanos), Lecco (Dr. Lusenti), Milano Niguarda (Dr.ssa Levati), Milano Policlinico, Milano San Raffaele, Monza Nuovo San Gerardo, Pavia 2a Rianimazione (Dr. Bellinzona), Roma Gemelli (Dr. Della Corte), Sondalo (Dr.ssa Ferraris), Torino CTO (Dr. Berardino), Treviso (Dr. Bosco), Trieste Gattinara (Dr. Viviani), Varese (Dr. Severgnini), Vicenza (Dr. Barbacini).

\section{Appendix. Database Design}

The data collection form is structured in six computer layouts:

\section{General/Demographic Data}

The first layout contains the data collected on the day of trauma and includes patient's details (age, sex, address, phone number) and information on time of injury, time of admission to the first emergency room and to the neurosurgical hospital. Type of admission is classified as "direct" to the neurosurgical hospital or "transfer". We also gather data about the type and mechanism of injury. The following fields are coded: pedestrian, road traffic accident, work, assault, domestic and other (causes not previously described). This epidemiological information will serve to establish the principal causes of traumatic lesion in Italy. We also notify the presence and type of extracranial injuries such as: hemorrhage (need for transfusion of at least two blood units), major chest injury, pneumothorax (requiring chest tube insertion), major abdominal injury, major limb injury, major facial injury, major spinal injury. A "major" lesion is classified as a severe enough to require hospitalisation in its own right.

\section{Pre-Admission and Admission Data}

This section collects quantitative information on insults, which may cause secondary brain damage. The patient's clinical situation is an evolving process and we have set three observational moments:

1. Pre-neurosurgical hospital admission (pre-NSHA). Information is collected on all the events that occurred before the patient arrived in the NICU. This period comprises the pre-hospital phase and peripheral hospital data, if the patient has been transferred to the Neurosurgical Hospital.

2. Neurosurgical Hospital Admission: reliable information to define the patient's condition on arrival in the neurosurgical hospital.

3. Post-stabilization. Unstable systemic conditions such as hypotension and hypoxia can negatively influence objective neurological assessment. The injured patient must therefore be evaluated once oxygenation, ventilation and perfusion have been restored. We define "Post-stabilization" as the moment when BP and oxygenation have been corrected and are within the physiological range. In sporadic cases, where a return to physiological values is not possible, we collect the best data during the first 24 hours.

In this layout (pre and admission data) numerical data on $\mathrm{Sa}_{2}$, $\mathrm{PaO}_{2}$, maximal and minimal $\mathrm{PaCO}_{2}, \mathrm{BP}, \mathrm{pH}$, haemoglobin and temperature are collected separately. Also indicated are the presence of an endotracheal tube and mechanical ventilation on arrival at the neurosurgical hospital.
Neurological data, the key to classification of the head injured patient, include pupil's size and reactivity and Glasgow Coma Score (GCS). The components of the GCS (motor response, verbal, and eye opening) are collected separately and the sum is displayed automatically.

The coded possibilities for pupillary response are bilaterally reactive pupils, unilaterally dilated unreactive pupil and bilaterally dilated unreactive pupils. This summary neuro-evaluation is simple and easily performed but at the same time contains all the information needed for classifying the patient and for identifying severe intracranial conditions such as brain herniation.

All this information is collected separately at the three time points: pre-neurosurgical hospital, at neurosurgical hospital admission and post-stabilization.

We also include a pre-trauma health evaluation that may influence the patient's outcome, summarized by the chronic section of the Apache I health evaluation score [5].

\section{Secondary Insults, Complications, Surgery}

This section identifies data relevant to different phases of the clinical history including:

1. Therapy pre neurosurgical hospital admission. This refers to therapy and manoeuvres performed with the aim of reducing ICP (even if ICP is not yet monitored in the pre-NSHA phase) and for transporting the traumatized patient. The coded fields are: proper sedation, mannitol, proper muscle relaxation, steroids and vasopressors (used for systemic hypotension). This information about preNSHA therapy will enable us to survey clinical practice in peripheral hospital and to evaluate the application of treatment guidelines.

2. First 24-h secondary insults. Clinical summary of insults in the first 24 hours after trauma. This enables us to collect more information than in the previous layout, where the data were quantitative but limited to the early phase. The aim of this section is to quantify any secondary insults in the period when these patients are most vulnerable to secondary brain damage.

Hypoxia. The coded possibilities are: no hypoxic episode, clinical hypoxic episode (cyanosis or bradypnea or metabolic acidosis $(\mathrm{pH}<7.25)$ or no airway protection), definite hypoxic episode (verified hypoxia with $\mathrm{Pa}_{2}<60 \mathrm{mmHg}$ or $\mathrm{Sp}_{2}<90 \%$ )

Hypotension: no hypotensive episode, clinical hypotensive episode (peripheral cyanosis or absence of peripheral pulse), definite hypotensive episode (systolic $\mathrm{BP}<95 \mathrm{mmHg}$ ).

Hypothermia: external temperature $<35.8^{\circ} \mathrm{C}$.

Early seizures: the possibilities are no seizures, suspected seizures (described by paramedical personnel or bystanders) or documented seizures (described by medical personnel in the clinical record).

3. Surgical intervention in the first 24 hours.

Extracranial and intracranial surgery performed in the first day. If intracranial surgery was needed we list the possibilities for such intervention: intracranial pressure monitoring placement, evacuation of extradural or subdural haematoma, removal of contusion, external decompression and other types of surgery.

4. ICU complications

Complications occurring during the ICU stay, requiring specific treatment, classified as:

a.) Intracranial complications such as delayed haematoma, ventriculitis/meningitis $\left[>100\right.$ cells $/ \mathrm{mm}^{3}$, reduced glucose $(<50 \%$ of the patient's current blood glucose) and elevated proteins $(>100 \mathrm{mg} / \mathrm{dl})$ ], seizures, raised intracranial pressure (ICP $>20 \mathrm{mmHg}$ for at least 15 minutes), intractable ICP 
(ICP $>30 \mathrm{mmHg}$ for more than 30 minutes) despite maximal therapy to reduce the ICP, brain herniation.

b.) Systemic complications such as respiratory failure, severe hypoxia $\left(\mathrm{PaO}_{2} / \mathrm{FiO}_{2}\right.$ ratio $\left.<150\right)$ and cardiovascular.

c.) Metabolic complications such as hyponatremia (sodium $<135 \mathrm{mEq} / \mathrm{l}$ ), diabetes insipidus (polyuria, urinary osmolarity less than $200 \mathrm{mOsm} / \mathrm{kg}$ ), hyperglycemia (blood glucose $>180 \mathrm{mg} / \mathrm{dl}$ )

d.) Infections: pneumonia (fever, leucocytosis, new infiltrates in the chest x-ray), urinary infection (fever, colonies $>10^{6}$ and/ or pyuria) and sepsis as defined by the Consensus Conference ACCP/SCCM in 1992 [1].

\section{CT Scan Data}

Two CT scans are selected for each patient: the first one and the worst during the first 15 days in the ICU. Information is collected separately for the two.

Each CT scan information is collected in a separate field: perimesencephalic cisterns (normal, compressed-absent), midline shift ( $<5 \mathrm{~mm}$ or $\geq 5 \mathrm{~mm}$ ), subarachnoid haemorrhage, pneumocephalus, hydrocephalus, hypodense lesions. The volumes of the two largest lesions are automatically computed by Cavalieri's direct estimator. The sum of the lesion volumes is displayed in a different field. Lesions are classified as intracerebral, extradural, and subdural. This coding system serves to classify all patients using Marshall's classification [8], on, subsequently, applying a new classification system on the raw data.

\section{Monitoring Information}

"Cerebral" monitoring used at least once during the ICU period. Details are required for ICP and jugular vein saturation monitoring (duration, type of catheter, side, and early and late complications). For ICP we collect information on length of monitoring (days), type of catheter and complications such as haemorrhage or infection. For jugular vein saturation monitoring we report the monitoring duration, type of catheter, side, early (carotid puncture, pneumothorax, increased ICP, malpositioning) and late complications (infections, thrombosis).

\section{Discharge and Outcome Information}

The last layout collects ICU discharge data, date and cause of death if applicable. A six months follow-up, using the Glasgow Outcome Score, is done for all the patients, by phone interview or medical visit.

\section{References}

1. American College of Chest Physicians/Society of Critical Care Medicine Consensus Conference (1992) Definitions for sepsis and organ failure and guidelines for the use of innovative therapies in sepsis. Crit Care Med 20: 6 864-874

2. Clatterbuck RE, Sipos EP (1997) The efficient calculation of neurosurgically relevant volumes from computed tomographic scans using Cavalieri's Direct Estimator. Neurosurgery 40: 339342

3. Foulkes MA, Eisenberg HM, Jane JA, Marmarou A, Marshall LF et al (1991) The traumatic coma data bank: design, methods and baseline characteristics. J Neurosurgery 75: S 8-13

4. Jennet B, Teasdale G, Galbraith S et al (1977) Severe head injuries in three countries. J Neurol Neurosurg Psychiatry 40: 291298

5. Knaus WA, Zimmerman JE, Wagner DP et al (1981) Apacheacute physiology and chronic health evaluation: a physiologically based classification system. Crit Care Med 9: 591-597

6. Marshall LF, Maas AI, Marshall SB et al (1998) A multicenter trial on the efficacy of using tirilazad mesylate in cases of head injury. J Neurosurg 89: 519-525

7. Marshall LF, Marshall SB, Klauber MR et al (1991) A new classification of head injury based on computerized tomography. $\mathbf{J}$ Neurosurg 75: s 14-20

8. Murray GD, Teasdale GM, Braakman R et al (1999) The European Brain Injury Consortium survey of head injuries. Acta Neurochir (Wien) 141: 223-236

9. Teasdale GM et al (1997) The European Brain Injury Consortium. Acta Neurochir (Wien) 139: 797-803

Correspondence: Dr. Citerio Giuseppe, Rianimazione, Nuovo H San Gerardo, via Donizetti, 106, 20052 Monza (MI) Italy. 